\title{
Activation of locus coeruleus heme oxygenase-carbon monoxide pathway promoted an anxiolytic-like effect in rats
}

\author{
P.G. Carvalho-Costa ${ }^{1,2}$, L.G.S. Branco ${ }^{2}$ and C.R.A. Leite-Panissi $i^{1,2}$ \\ ${ }^{1}$ Programa de Pós-Graduação em Psicobiologia, Faculdade de Filosofia, Ciências e Letras de Ribeirão Preto, \\ Universidade de São Paulo, Ribeirão Preto, SP, Brasil \\ ${ }^{2}$ Departamento de Morfologia, Fisiologia e Patologia Básica, Faculdade de Odontologia de Ribeirão Preto, \\ Universidade de São Paulo, Ribeirão Preto, SP, Brasil
}

\begin{abstract}
The heme oxygenase-carbon monoxide pathway has been shown to play an important role in many physiological processes and is capable of altering nociception modulation in the nervous system by stimulating soluble guanylate cyclase (sGC). In the central nervous system, the locus coeruleus (LC) is known to be a region that expresses the heme oxygenase enzyme (HO), which catalyzes the metabolism of heme to carbon monoxide (CO). Additionally, several lines of evidence have suggested that the LC can be involved in the modulation of emotional states such as fear and anxiety. The purpose of this investigation was to evaluate the activation of the heme oxygenase-carbon monoxide pathway in the LC in the modulation of anxiety by using the elevated plus maze test (EPM) and light-dark box test (LDB) in rats. Experiments were performed on adult male Wistar rats weighing 250-300 g ( $\mathrm{n}=182)$. The results showed that the intra-LC microinjection of heme-lysinate (600 nmol), a substrate for the enzyme $\mathrm{HO}$, increased the number of entries into the open arms and the percentage of time spent in open arms in the elevated plus maze test, indicating a decrease in anxiety. Additionally, in the LDB test, intra-LC administration of heme-lysinate promoted an increase on time spent in the light compartment of the box. The intracerebroventricular microinjection of guanylate cyclase, an SGC inhibitor followed by the intra-LC microinjection of the heme-lysinate blocked the anxiolytic-like reaction on the EPM test and LDB test. It can therefore be concluded that $\mathrm{CO}$ in the LC produced by the HO pathway and acting via cGMP plays an anxiolytic-like role in the LC of rats.
\end{abstract}

Key words: Heme oxygenase; Carbon monoxide; Locus coeruleus; Anxiety; Elevated plus maze, Light/dark box

\section{Introduction}

The exposure of animals to dangerous situations is potentially effective in eliciting responses characteristic of fear and anxiety. Because there appears to be a correlation between human defensive behavior and fear- and anxietyrelated defensive patterns in non-human mammals (1), many studies have used animal models to study the neural substrates involved in the modulation of fear and anxiety in humans. In particular, the elevated plus maze (EPM) is an animal model where the behavioral repertoire of rodents is used to detect effects on anxiety, and it has been proven to be bidirectionally sensitive to manipulations of anxiety (2).

It has been established that the neural substrates responsible for the modulation of defensive behaviors, aversive reactions, and emotional states fundamentally consist of the amygdala, hypothalamus, and periaqueductal gray matter. However, other structures are also related to the expression of the emotional responses; in particular, the noradrenergic system of the locus coeruleus (LC), which is a system closely correlated with attentional states, sleep/wakefulness cycles, learning and memory, reproduction, emotional behaviors and stressful situations, as well as modulation of fear and anxiety (3). In fact, stressful situations, including negative emotions, such as anxiety and/or fear, promote an increase in noradrenaline release in distinct brain areas, in particular in the hypothalamus, amygdala, and LC (4).

Neurochemistry research has shown that various systems of neurotransmitters are involved in fear and anxiety modulation, such as gamma-aminobutyric acid (GABA), serotonin, norepinephrine and glutamate $(5,6)$. In addition, a growing number of studies have given support that nitric oxide (NO) and carbon monoxide (CO) can modulate emotional and autonomic responses related with stress $(6,7)$. In this way, both $\mathrm{CO}$ and $\mathrm{NO}$ exert 
a stimulatory influence on the acute adrenocorticotropic hormone $(\mathrm{ACTH})$ response to physical-emotional stressors. $\mathrm{CO}$ and NO share similar properties that can activate soluble guanylate cyclase (sGC), resulting in the complex regulation of 3'-5'-guanosine monophosphate (cGMP) $(8,9)$. Likewise, Quock and Nguyen (10) demonstrated that systemic pretreatment with L-NOARG (an inhibitor of NO synthase) is able to block the anxiolytic effect of chlordiazepoxide in mice submitted to the EPM, indicating that NO may exert an anxiolytic effect in mice.

The activity of $\mathrm{CO}$ depends on the heme oxygenase (HO) enzyme that catalyzes the conversion of heme to $\mathrm{CO}$, heme iron, and biliverdin (9). Two forms of $\mathrm{HO}, \mathrm{HO}-1$ and $\mathrm{HO}-2$, have been identified, and a third form, $\mathrm{HO}-3$, may be present in rats $(11,12)$. In the central and peripheral nervous systems, $\mathrm{HO}-2$ seems to be responsible for most of the $\mathrm{HO}$ activity and is expressed in both glial and neuronal cells (13), as well as cell layers in the olfactory bulb, hippocampus, cerebellum, spinal cord, and LC (9). It is important to note that LC displays elevated expression of $\mathrm{HO}-2$, whereas the expression of nitric oxide synthase (responsible for the NO formation) is lower $(9,14)$. Thus, it is suggested that CO participates in the modulation of the functions performed by this structure. Previous studies have demonstrated that the endogenous HO-CO-cGMP pathway in the LC is critical to modulation of thermal response during development of endotoxin fever, as well as during hypothermic response to restraintstress $(7,15)$. However, the role of the HO-CO-cGMP pathway in the other functions modulated by the LC remains to be clarified, including its role in emotional behavior. Furthermore, previous studies have demonstrated a marked increase of c-fos expression (16) and HO-2 expression (9) in LC after stressful stimuli. Therefore, the present study was designed to investigate whether the HO-CO pathway of the LC can modulate emotional behavior. To this end, we investigated whether the microinjection of zinc deuteroporphyrin 2,4-bis glycol (ZnDPBG, an $\mathrm{HO}$ inhibitor), heme-lysinate (substrate overload) or the selective inhibitor of soluble guanylate cyclase $(1 \mathrm{H}-(1,2,4)$ oxadiazolo [4,3-a] quinoxaline-1-one (ODQ)) into the LC in different groups of rats produces alterations in emotional behavior as assessed by the elevated plus maze test (EPM) and light-dark box test (LDB) in rats.

\section{Material and Methods}

\section{Animals \\ Experiments were performed on adult male Wistar rats weighing 250-300 g, ( $n=182)$ obtained from the animal facility of the Universidade de São Paulo, Ribeirão Preto, $\mathrm{SP}$, Brazil. Animals were housed in a temperature- controlled room $\left(24 \pm 1^{\circ} \mathrm{C}\right)$ and in a 12-h light/dark cycle (lights on at 6:00 am) with food and water ad libitum. The experiments were carried out in compliance with the}

recommendations of the Sociedade Brasileira de Ciência em Animais de Laboratório and with the approval of the Animal Care and Use Committee of the Universidade de São Paulo, Brazil at the Ribeirão Preto campus (Protocol \#09.1.606.53.7). All efforts were made to minimize animal suffering.

\section{Surgical procedures}

The rats were anesthetized by intramuscular injection of ketamine $(100 \mathrm{mg} / \mathrm{kg}$, União Química Farmacêutica Nacional S.A., Brazil) plus xylazine $(10 \mathrm{mg} / \mathrm{kg}$, Calier S.A., Spain) and placed in a stereotaxic apparatus (David-Kopf Instruments, USA) with the incisive bar at $0 \mathrm{~mm}$ and inclination of vertical stereotaxic bar at $15^{\circ}(17)$. One stainless-steel guide cannula $(0.6 \mathrm{~mm}$ o.d. and $15 \mathrm{~mm}$ in length) was inserted $1 \mathrm{~mm}$ above the right $L C$ region (coordinates: anterior: $3.4 \mathrm{~mm}$ from lambda, lateral: $1.2 \mathrm{~mm}$ from midline, dorsal: $5.8 \mathrm{~mm}$ from the skull surface). As one of the experiments required two microinjections, animals designated to be used in this experiment were implanted with a second cannula ( $0.6 \mathrm{~mm}$ o.d. and $12 \mathrm{~mm}$ in length) in the right lateral cerebral ventricle (coordinates: anterior $-1.0 \mathrm{~mm}$, lateral $-1.6 \mathrm{~mm}$ from midline, dorsal 3.2 to $3.7 \mathrm{~mm}$ from the skull surface); incisive bar at $-3.3 \mathrm{~mm}$. The displacement of the meniscus in a water manometer ensured correct positioning of the cannula in the lateral ventricle. The cannulas were fixed to the skull by means of self-polymerizing resin and an additional anchoring screw. A tight-fitting stylet was kept inside the guide cannula to prevent occlusion. After surgery, the rats received a subcutaneous injection of the anti-inflammatory and analgesic Banamine (10 mg/mL flunixin meglumine; Schering-Plough, USA) and an antibiotic (benzyl penicillin 160,000 U/kg, Fort Dodge, Brazil, administered intramuscularly). The experiments were initiated 1 week after surgery.

\section{Drugs and microinjection procedure}

The non-selective $\mathrm{HO}$ inhibitor ZnDPBG, at the doses 5,50 and $200 \mathrm{nmol} / 0.1 \mu \mathrm{L}$ ) and hemin were obtained from Porphyrin Products (USA). ZnDPBG was dissolved in $50 \mathrm{mM} / \mathrm{L}$ of $\mathrm{Na}_{2} \mathrm{CO}_{3}$ and stored in the dark. Hemin was used to prepare heme-lysinate (at the doses 150, 300 and $600 \mathrm{nmol} / 0.1 \mu \mathrm{L})$, a HO-CO-cGMP pathway inducer. Heme-free preparations were used as amino acid (L-lysine) vehicle control solutions. The vehicle of hemelysinate consisted of L-lysine $(14.2 \mu \mathrm{mol} / \mathrm{mL})$, ethanol (5\%), propylene glycol $(40 \%)$ and sterile water $(55 \%)$. The sGC inhibitor $1 \mathrm{H}-(1,2,4)$ oxadiazolo [4,3-a] quinoxaline-1one (ODQ, $1.3 \mathrm{nmol} / 1.0 \mu \mathrm{L}$ ) was purchased from Tocris Cookson (USA) and dissolved in a vehicle consisting of $1 \%$ DMSO in pyrogen-free sterile saline $(7,18)$. These doses were based on previous studies $(7,15)$.

A $10-\mu \mathrm{L}$ Hamilton syringe and a dental injection needle connected to a PE-10 tube were used to perform the microinjections into the LC of conscious rats. The injection needle was $1 \mathrm{~mm}$ longer than the guide cannula so that 
the LC was reached by the needle only at the time of injection. The microinjections were performed at a rate of $0.1 \mu \mathrm{L}$ over a period of one minute. To prevent reflux, the needle was kept inside the guide cannula for $40 \mathrm{~s}$ after the end of the infusion.

\section{Elevated plus maze test (EPM)}

EPM test was used to assess the anxiety levels of the rats. The EPM was made of wood, and consisting of two open arms $(50 \times 10 \mathrm{~cm})$ crossed at right angles with two closed arms of the same size, according to the specifications described in Morato and Brandão (19). The two closed arms were enclosed by walls $50 \mathrm{~cm}$ high, with he exception of the central part of the maze $(10 \times 10 \mathrm{~cm})$ where the open and closed arms crossed. The entire EPM was elevated $50 \mathrm{~cm}$ above the floor and the luminosity at the level of the EPM open arms was 30 lux. It is important to note that rats naturally avoid threatening situations represented in the model by the height and open space (19). Behavior in the EPM was recorded by a video camera linked to a monitor. This device, located outside the experimental room, allowed the recordings to be analyzed later. Rats were placed individually in the center of the maze facing a closed arm and allowed to explore the maze for a 5-min test period. EPM measures (number of entries and time spent in each arm) were recorded as described in Pellow et al. (20). The open-arm activity was evaluated as the time spent in the open arms relative to the total time spent in the plus maze (300 s), and was expressed as a percentage. At the end of the session, the rat was returned to its home cage, and before the next rat was tested, the maze was wiped clean with a $70 \%$ alcohol solution and dried with paper towels.

\section{Light-dark box test (LDB)}

The LDB consisted of two compartments, a dark area (30 cm length $\times 80 \mathrm{~cm}$ width $\times 60 \mathrm{~cm}$ height) and a light area $(50 \mathrm{~cm}$ length $\times 80 \mathrm{~cm}$ width $\times 60 \mathrm{~cm}$ height) connected by an opening ( $15 \mathrm{~cm}$ height $\times 10 \mathrm{~cm}$ width). The box was made of plastic. The light intensity in the dark side was less than 5 lux. The light region was uncovered on the top and received room light. Rodents, which are nocturnal animals, have a natural tendency to spend more time in the dark compartment, because light places represent a natural threat. Animals were placed in the light side of the chamber facing the opening to the dark chamber and allowed to move freely between the two compartments for $5 \mathrm{~min}$ sessions. Behaviors were videotaped and scored using Geo Vision software. The parameters analyzed were the following: number of transitions (the number of dark compartment to light compartment transitions) and the total time spent in the light and in the dark compartments (21). An animal was considered to be in one of the compartments when its head and front paws were in that area of the box. At the end of the session, animals were returned to their home cages, and the area was wiped clean with a $70 \%$ alcohol solution.

\section{Experimental protocols}

Effects of intra- $L C$ injection of ZnDPBG. Rats that were previously cannulated were microinjected intra-LC with the $\mathrm{HO}$ inhibitor ZnDPBG (5, 50 or $200 \mathrm{nmol}, 0.1 \mu \mathrm{L} ; \mathrm{n}=8$ ) or with the vehicle $\left(\mathrm{Na}_{2} \mathrm{CO}_{3}, 50 \mathrm{nmol}, 0.1 \mu \mathrm{L} ; \mathrm{n}=6\right)$. The rats were submitted 15 min later to the EPM or the LDB test for a period of $5 \mathrm{~min}$.

Effects of intra-LC injection of heme-lysinate. Rats that were previously cannulated were microinjected intra-LC with the $\mathrm{HO}$ substrate, heme-lysinate $(150,300$ or $600 \mathrm{nmol}$, $0.1 \mu \mathrm{L} ; \mathrm{n}=8)$, or with the L-lysine vehicle solution $(0.1 \mu \mathrm{L} ; \mathrm{n}=8)$. The rats were submitted $15 \mathrm{~min}$ later to the EPM or the LDB test for a period of $5 \mathrm{~min}$.

Effects of intra-LC injection of $S G C$ inhibitor (ODQ). Rats that were previously cannulated received intracerebroventricular (icv) microinjections of the sGC inhibitor ODQ (1.3 nmol, $1.0 \mu \mathrm{L} ; \mathrm{n}=8$ ) or of the vehicle (DMSO $1 \%$, $1.0 \mu \mathrm{L} ; \mathrm{n}=5)$ followed by the intra-LC microinjection of the heme-lysinate $(600 \mathrm{nmol}, 0.1 \mu \mathrm{L} ; \mathrm{n}=8)$ or the vehicle (L-lysine, $14.2 \mu \mathrm{mol}, 0.1 \mu \mathrm{L} ; \mathrm{n}=8$ ). ODQ or its vehicle was injected in an icv manner to avoid multiple injections into the LC, which could eventually cause lesions in the nucleus. This method was based on a previous study (7). The rats were submitted 15 min later to the EPM or the LDB test for a period of $5 \mathrm{~min}$.

\section{Histology}

At the end of the experiments, rats were anesthetized with an intramuscular injection of ketamine $(225 \mathrm{mg} / \mathrm{kg}$, União Química Farmacêutica Nacional S.A., Brazil) plus xylasine (30 mg/kg, Carlier S.A., Spain), and transcardially perfused with saline ( $\mathrm{NaCl}, 0.9 \%)$, followed by $10 \%$ formalin. The brains were then removed and fixed in $10 \%$ formalin for 4 days. The tissue was submitted to routine histological processing, and sections were observed under a microscope (Carl Zeiss model KS300, Germany) to determine the locations of the stimulated sites using the Paxinos and Watson Atlas (17).

\section{Statistical analysis}

Results were first submitted to Levene's test for homogeneity of variance. One-way analysis of variance (ANOVA) was then used for each parameter analyzed, followed by the post hoc Newman-Keuls test. Data were considered statistically significant when $P<0.05$. Results are reported as means \pm SE.

\section{Results}

Figure 1 shows a representative photomicrograph of the unilateral intra-LC microinjection. The microinjected area corresponds to the compact cluster of neurons adjacent to the fourth ventricle in the pontine brainstem, 


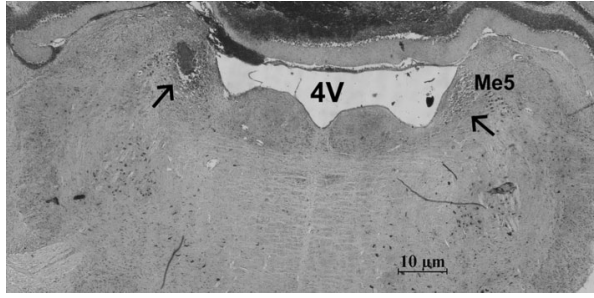

Figure 1. Representative photomicrograph of the right locus coeruleus (LC) of the rat. The figure shows a coronal section at the pons level illustrating the location of the intact LC (right arrow) and a typical intra-LC microinjection (left arrow). 4V: fourth ventricle; Me5: mesencephalic trigeminal nucleus.

according to the atlas of Paxinos and Watson (17). PeriLC microinjections caused no apparent change in EPM and LDB measures, in comparison to rats treated with vehicle in all experimental groups (data not shown).

At all doses, the intra-LC administration of ZnDPBG did not alter the percentage of time in the open arms $(F=2.21, P=0.109)$, the mean number of entries into open arms $(F=1.91, P=0.150)$, or the number of entries into closed arms ( $F=1.06, P=0.382)$ for rats in the EPM test (Figure 2A-C). Regarding the LDB test, intra-LC administration of ZnDPBG did not alter the time spent in the light compartment $(F=1.858, P=0.161$, Figure $2 D)$, or the number of transitions in the LDB $(F=0.456, P=0.715$, Figure 2E).

The results of this study revealed an increase in the percentage of time spent in and entries into the open arms of the EPM by rats that received intra-LC microinjections of heme-lysinate ( $600 \mathrm{nmol}$, Figure $3 \mathrm{~A}$ and $\mathrm{B})$. Considering the percentage of time spent in the open arms, a significant increase between treatments was found $(F=10.73, P<0.001)$ when comparing the $600 \mathrm{nmol}$ Heme-lys group with other groups $(P<0.05$, Figure $3 A)$. Regarding the mean entries into open arms, a significant increase was observed $(F=20.69, P<0.001)$ when the $600 \mathrm{nmol}$ Heme-lys group was compared with Lys and the $150 \mathrm{nmol}$ and $300 \mathrm{nmol}$ Heme-Lys groups (Figure 3B). Finally, the mean entries into closed arms of the EPM did not vary between treatments (Figure $3 \mathrm{C}$ ). Regarding the LDB test, a significant increase was found $(F=25.43$, $\mathrm{P}<0.001)$ in the time spent in the light compartment for the Heme-Lys groups $(150,300$ and $600 \mathrm{nmol})$ when compared with the Lys group (Figure 3D). However, Heme-Lys microinjected intra-LC, at all doses, did not alter the number of transitions of rats in the LDB (Figure 3E).

The results reported in Figure 4 show that the icv microinjection of ODQ, an $\mathrm{SGC}$ inhibitor, blocked the increase in the percentage of time spent in, and entries into, the open arms of the EPM induced by intra-LC microinjections of heme-lysinate $(600 \mathrm{nmol})$. Considering the percentage of time spent in the open arms, significant differences were found in the behaviors $(F=61.65$, $P=0.002$ ) when comparing the $O D Q+$ Heme group with other groups $(P<0.05$, Figure $4 A)$. Nevertheless, regarding the mean entries into the open arms, significant increases were observed $(F=30.18, P<0.001)$ when comparing the $\mathrm{ODQ}+$ Heme group with the DMSO+ L-lysine, $O D Q+L-l y s i n e$ and $O D Q+$ Heme groups (Figure 4B). Moreover, the mean number of entries into

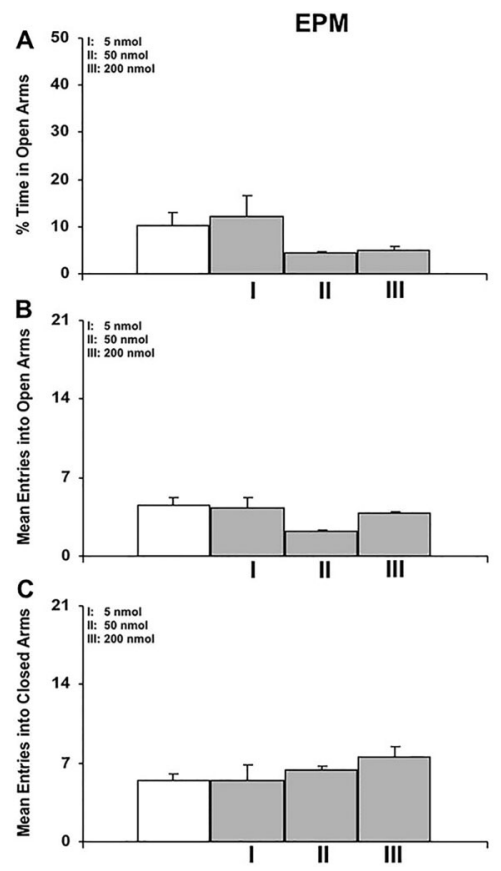

LDB
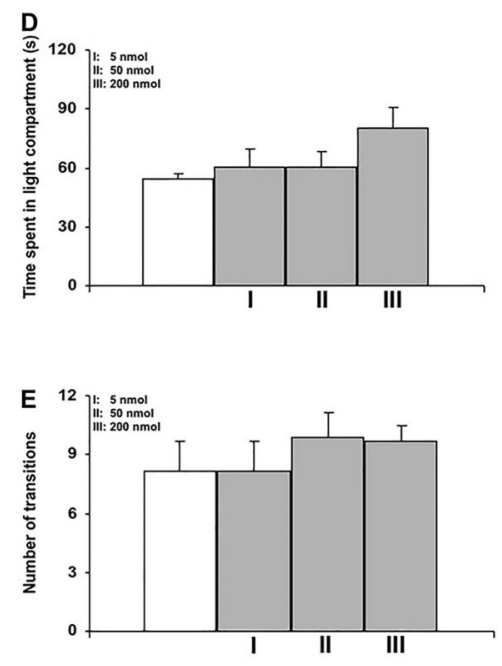

$\square \mathrm{Na}_{2} \mathrm{CO}_{3} \quad \square$ ZnDPBG
Figure 2. Intra-locus coeruleus administration of the nonspecific inhibitor of the enzyme heme oxygenase ( $\mathrm{HO}$ ), ZnDPBG (5, 50 and $200 \mathrm{nmol}$ ), or its vehicle $\left(\mathrm{Na}_{2} \mathrm{CO}_{3}\right)$ did not alter the percentage of time spent in the open arms $(A)$, the mean number of entries into open $(B)$ and closed arms $(C)$ in the elevated plus maze (EPM) test, and on time spent in the light compartment $(D)$ and number of transitions $(E)$ in the light-dark box (LDB) test. One-way ANOVA was used for statistical analysis. Data are reported as means \pm SE $(n=6-8$ animals per group). 

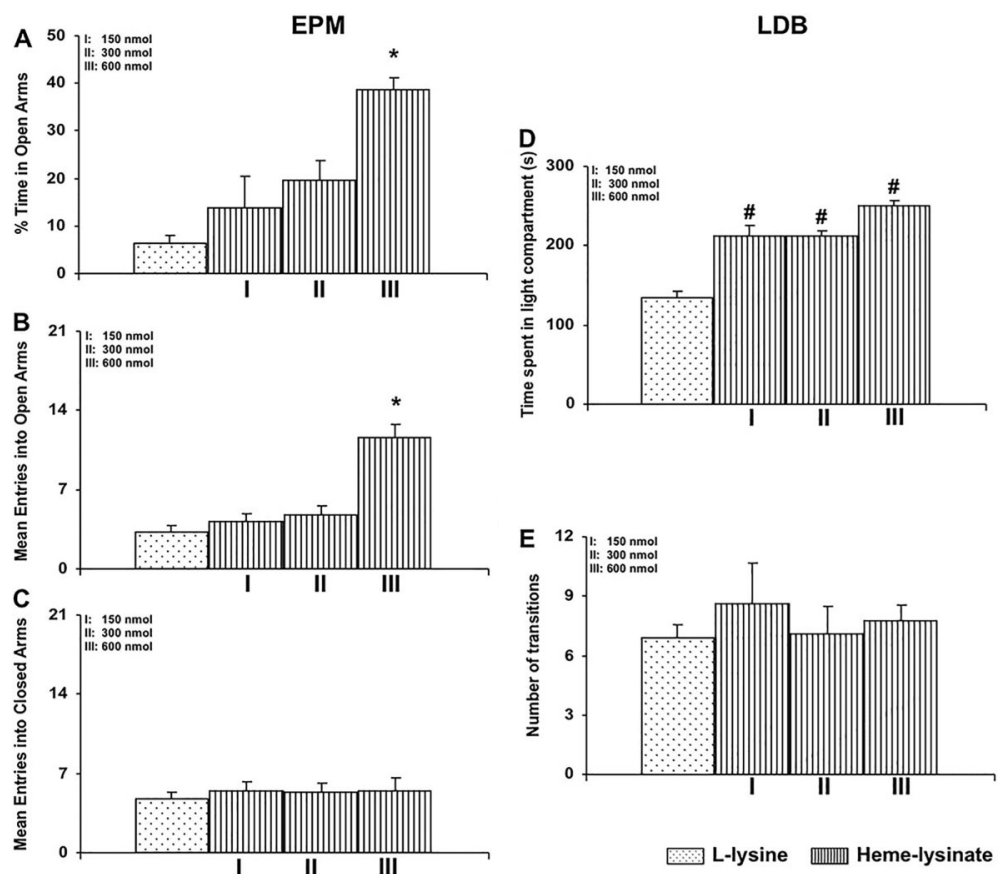

Figure 3. Effects of intra-locus coeruleus administration of the substrate, heme-lysinate $(150,300$ and $600 \mathrm{nmol}$ ), or its vehicle (L-lysine) on the percentage of time spent in the open arms $(A)$, the mean number of entries into open $(B)$ and closed arms $(C)$ in the elevated plus maze (EPM) test, and on time spent in the light compartment $(D)$ and number of transitions $(E)$ in the light-dark box (LDB) test. Data are reported as means $\pm \mathrm{SE}$. ${ }^{*} \mathrm{P}<0.05$, compared with its respective control (L-lysine) and with the 150 and $300 \mathrm{nmol}$ hemelysinate groups; ${ }^{*} \mathrm{P}<0.05$, compared with its respective control (L-lysine) ( $\mathrm{n}=8$ animals per group) (Newman-Keuls test). the closed arms of the EPM did not vary between treatments (Figure 4C). Additionally, pre-treatment with ODQ (icv) blocked the increase in time spent in the light compartment $(F=115.42, P<0.001)$ induced by the intraLC microinjection of DMSO + Heme (600 nmol, Figure 4D) when compared with the DMSO + L-lysine, ODQ + L-lysine and $\mathrm{ODQ}+$ Heme groups (Figure 4D). Finally, none of the treatments altered the number of transitions in the LDB (Figure 4E).

\section{Discussion}

The results of the present study indicated that the activity of the $\mathrm{HO}-\mathrm{CO}$ pathway in the locus coeruleus can
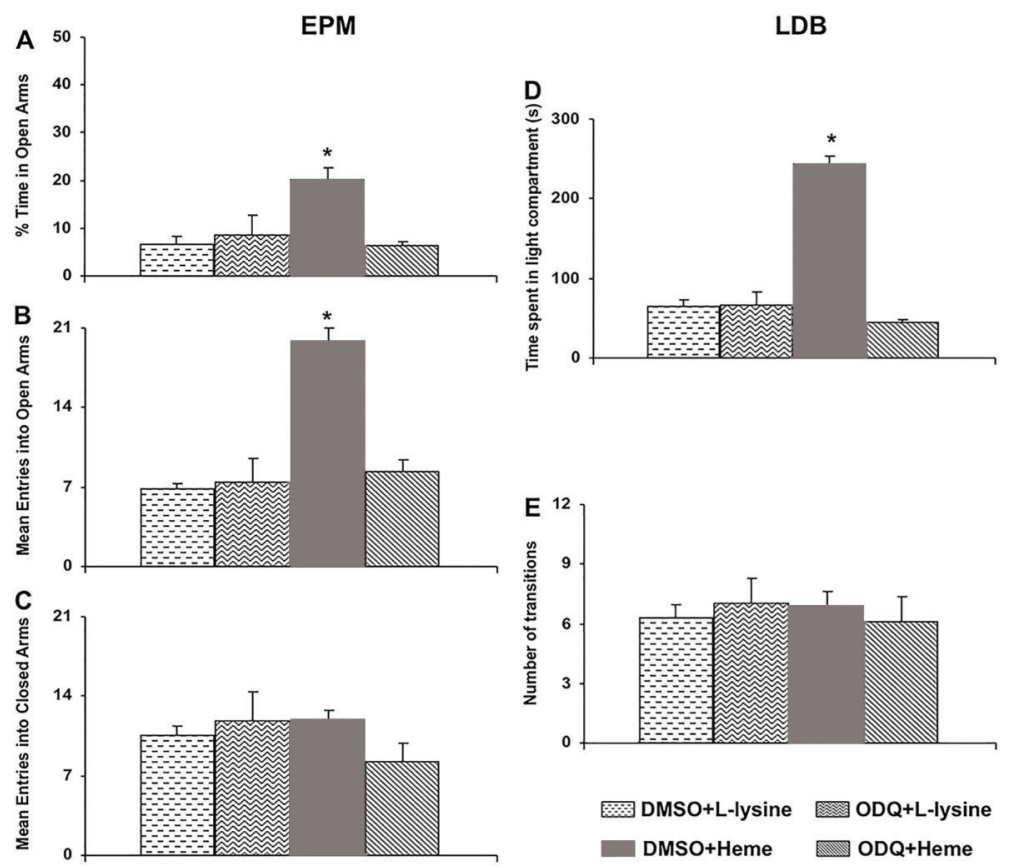

Figure 4. Effects of $i c v$ administration of $1 \mathrm{H}-(1,2,4)$ oxadiazolo [4,3-a] quinoxaline-1-one (ODQ) $(1.3$ $\mathrm{nmol}$, a soluble guanylate cyclase inhibitor) or its vehicle ( $1 \%$ DMSO) and intra-locus coeruleus administration of the heme-lysinate $(600 \mathrm{nmol})$ or its vehicle (L-lysine) on the percentage of time spent in the open arms $(A)$, the mean number of entries into open $(B)$ and closed arms $(C)$ in the elevated plus maze (EPM) test, and on time spent in the light compartment $(D)$ and number of transitions $(E)$ in the light-dark box (LDB) test. Data are reported as means $\pm S E$. ${ }^{*} P<0.05$ compared to other groups ( $n=5-8$ animals per group) (Newman-Keuls test). 
modulate anxiety, as assessed by the EPM test and the LDB test in rats. In particular, it was observed that the facilitation of enzymatic action by the administration of its substrate, heme-lysinate (at the dose of $600 \mathrm{nmol}$ ), promoted an increase in the number of open arm entries and the percentage of time spent in open arms in the EPM test. In a similar way, in the LDB test, it was observed that the facilitation of enzymatic action by the administration of its substrate, heme-lysinate (at the dose of $600 \mathrm{nmol}$ ) promoted an increase in time spent in the light compartment of the box. Taken together, these results suggest an anxiolytic effect of the HO-CO pathway in the LC. Additionally, the icv microinjection of the ODQ (an sGC inhibitor) followed by the intra-LC administration of hemelysinate $(600 \mathrm{nmol})$ blocked the anxiolytic-like effect on the EPM test and LDB test. It is important to note that CO has a physiological function similar to NO (9), and high levels of SGC are present in LC (8). However, NOS-like immunoreactivity is present in nuclei adjacent to LC (22). So, it is possible that increased activity of cGMP is mediated by the action of $\mathrm{CO}$ rather than by the action of NO in the LC (14).

There is strong evidence supporting the role of catecholaminergic LC neurons in emotional behaviors $(3,23)$. Results from functional studies show that robust activation of the LC is observed in cats submitted to stressful situations (24), which also caused an increased expression of c-fos in this region of mice (25). In particular, a previous study (4) suggested that negative emotions, such as fear and/or anxiety, increased noradrenergic activity in the hypothalamus, amygdala and LC. Furthermore, anatomical studies demonstrated that LC projects divergent efferent pathways to the forebrain, including hypothalamus (26) and amygdala (27), structures that are essential for emotional modulation (28).

Additionally, Khoshbouei et al. (29) demonstrated that amplifying the noradrenergic response to stress by means of yohimbine treatment prior to submission to acute stress released galanin in central nucleus of the amygdala, which produced an anxiolytic response in EPM test. Considering the results obtained in this study, it is possible that the intraLC administration of heme-lysinate increased the activity of the HO-CO pathway, which in turn could have promoted an increase of the firing rate of noradrenergic neurons of the LC (14). This could have caused an alteration of noradrenergic release in forebrain structures, such as hypothalamus and amygdala, resulting in an anxiolytic effect. Within this perspective, clinical findings suggest a relationship between the central noradrenergic system in fear/anxiety states and depression in humans. This suggestion is based on the fact that treatment with the $\alpha 2$-adrenergic agonist, clonidine, is effective in treating patients with anxiety disorders, whereas the administration of the $\alpha 2$-adrenergic antagonist exacerbates emotional symptoms (30). A previous study demonstrated that rats with chemical lesions on catecholaminergic neurons produced by a 6-hydroxydopamine injection in the LC showed normal motor activity, exploration, and habituation (23), suggesting that the LC is not essential for controlling motor activity. Corroborating previous findings, the results obtained in this study showed that heme-lysinate intra-LC administrated did not modify the mean number of entries into closed arms in EPM test, or the number of transitions in LDB test, which are parameters for locomotor behavior $(2,31)$. So, it is possible to suggest that the anxiolytic-like effect, evidenced by an increase in the number of open arms entries and the percentage of time in open arms in the EPM test, and an increase in time spent in the light compartment in the LDB test is due to emotional modulation rather than motor activity alteration.

The noradrenergic system has been identified as one of the important regulatory systems for the hypothalamicpituitary-adrenal axis, acting mainly on the release of corticotropin releasing factor in neurons in the paraventricular nucleus (PVN) of the hypothalamus (32). In addition, Ziegler et al. (33) showed that lesions in catecholaminergic neurons in the LC by the administration of 6-hydroxydopamine attenuated the release of ACTH in animals submitted to physical restraint stress. Considering the anatomical findings, it is possible that the activation of noradrenergic neurons of the LC promotes the inhibition of neurons in the prefrontal cortex and the disinhibition of GABAergic efferent neurons in this region, which leads to the activation of the PVN and, consequently, the activation of the hypothalamicpituitary-adrenal circuit (34). Furthermore, the involvement of the LC in modulating states of fear and anxiety could also occur by efferent projections to the basolateral nucleus of the amygdala, because the activity of neurons in the LC promotes their own inhibition through a negative feedback (35). However, the noradrenergic modulation in the basolateral amygdala seems to have different dose-dependent effects on anxiety behavior (36). In this way, Valizadegan et al. (36) showed that low doses of salbutamol (a $\beta$-adrenergic agonist) decreased the percentage of open arms time and open arms entries in the EPM test, indicating an anxiogenic effect, while the highest dose decreased the anxiety parameters.

Initial studies conducted by Redmond et al. (37) showed that electrical stimulation of the LC in monkeys resulted in behavior observed in situations of intense fear. However, in rats, studies have shown opposite results $(38,39)$. In particular, depletion of norepinephrine in rats resulted in increased fear and anxiety in novel places (38). On the other hand, chemical injury in the noradrenergic neurons of the LC promoted reduction of anxiogenic behaviors assessed with the EPM test, suggesting an anxiolytic effect after removing the influence of neurons in the LC (39). According to Weiss et al. (40), studies with animal models have suggested that the activity of the noradrenergic system in the LC is related to reduced anxiety and fear, contrary to a theory first described by Redmond et al. (37) in which the increased activity in 
neurons of the LC results in fear and anxiety. Therefore, it is clear that discrepancies exist and that more studies are still needed for a better understanding of the activity of neurons in the LC.

In summary, the results obtained in this study showed that the facilitation of enzymatic action by the administration of its substrate, heme-lysinate (at the dose of 600 $\mathrm{nmol}$ ), promoted an increase in the number of open arm entries and the percentage of time spent in open arms in the EPM test, and an increase in time spent in the light compartment in the LDB test. This effect was blocked by the icv administration of ODQ, a sGC inhibitor. These data suggest that $\mathrm{CO}$ in the LC produced by the $\mathrm{HO}$ pathway and acting via cGMP played an anxiolytic role. Perhaps this anxiolytic-like effect occurred because the altered

\section{References}

1. Blanchard DC, Griebel G, Blanchard RJ. Mouse defensive behaviors: pharmacological and behavioral assays for anxiety and panic. Neurosci Biobehav Rev 2001; 25: 205-218, doi: 10.1016/S0149-7634(01)00009-4.

2. Rodgers RJ, Cao BJ, Dalvi A, Holmes A. Animal models of anxiety: an ethological perspective. Braz J Med Biol Res 1997; 30: 289-304, doi: 10.1590/S0100-879X1997000300002.

3. Berridge CW, Waterhouse BD. The locus coeruleus-noradrenergic system: modulation of behavioral state and statedependent cognitive processes. Brain Res Brain Res Rev 2003; 42: 33-84, doi: 10.1016/S0165-0173(03)00143-7.

4. Tanaka $M$, Yoshida $M$, Emoto $H$, Ishii $H$. Noradrenaline systems in the hypothalamus, amygdala and locus coeruleus are involved in the provocation of anxiety: basic studies. Eur J Pharmacol 2000; 405: 397-406, doi: 10.1016/S00142999(00)00569-0.

5. Engin $E$, Treit $D$. The effects of intra-cerebral drug infusions on animals' unconditioned fear reactions: a systematic review. Prog Neuropsychopharmacol Biol Psychiatry 2008; 32: 1399-1419, doi: 10.1016/j.pnpbp.2008.03.020.

6. Kim CK, Rivier CL. Nitric oxide and carbon monoxide have a stimulatory role in the hypothalamic-pituitary-adrenal response to physico-emotional stressors in rats. Endocrinology 2000; 141: 2244-2253, doi: 10.1210/endo.141.6.7500.

7. Ravanelli MI, Branco LG. Role of locus coeruleus heme oxygenase-carbon monoxide-cGMP pathway during hypothermic response to restraint. Brain Res Bull 2008; 75: 526-532, doi: 10.1016/j.brainresbull.2007.09.002.

8. Furuyama T, Inagaki S, Takagi H. Localizations of alpha 1 and beta 1 subunits of soluble guanylate cyclase in the rat brain. Brain Res Mol Brain Res 1993; 20: 335-344, doi: 10.1016/0169-328X(93)90060-3.

9. Maines MD. The heme oxygenase system: a regulator of second messenger gases. Annu Rev Pharmacol Toxicol 1997; 37: 517-554, doi: 10.1146/annurev.pharmtox.37.1. 517.

10. Quock RM, Nguyen E. Possible involvement of nitric oxide in chlordiazepoxide-induced anxiolysis in mice. Life Sci 1992; 51: L255-L260, doi: 10.1016/0024-3205(92)90119-A.

11. McCoubrey WK Jr, Huang TJ, Maines MD. Isolation and characterization of a cDNA from the rat brain that encodes activity of the neurons of the LC, causing a potentiated noradrenergic tonus of this region, might be related to anxiety and fear modulation. Further studies are needed to clarify this involvement.

\section{Acknowledgments}

The authors would like to thank Patricia Adriana Basile for her technical assistance and Dr. Maria Ida Bonini Ravanelli for her histological analyses. This work was supported by CAPES/PROEX, FAPESP (\#2007/08122-7). P.G. Carvalho-Costa received a $\mathrm{PhD}$ scholarship from CAPES. L.G.S. Branco (\#300949/2013-8) and C.R.A. Leite-Panissi (\#07383/2012-1) received research grants from CNPq. hemoprotein heme oxygenase-3. Eur J Biochem 1997; 247: 725-732, doi: 10.1111/j.1432-1033.1997.00725.x.

12. Liang D, Li X, Lighthall G, Clark JD. Heme oxygenase type 2 modulates behavioral and molecular changes during chronic exposure to morphine. Neuroscience 2003; 121: 999-1005, doi: 10.1016/S0306-4522(03)00483-4.

13. Dwyer BE, Nishimura RN, Lu SY. Differential localization of heme oxygenase and NADPH-diaphorase in spinal cord neurons. Neuroreport 1995; 6: 973-976.

14. Pineda J, Kogan JH, Aghajanian GK. Nitric oxide and carbon monoxide activate locus coeruleus neurons through a cGMPdependent protein kinase: involvement of a nonselective cationic channel. J Neurosci 1996; 16: 1389-1399.

15. Ravanelli MI, Almeida MC, Branco LG. Role of the locus coeruleus carbon monoxide pathway in endotoxin fever in rats. Pflugers Arch 2007; 453: 471-476, doi: 10.1007/s00424-0060136-8.

16. Chowdhury GM, Fujioka T, Nakamura S. Induction and adaptation of Fos expression in the rat brain by two types of acute restraint stress. Brain Res Bull 2000; 52: 171-182, doi: 10.1016/S0361-9230(00)00231-8.

17. Paxinos G, Watson C. The rat brain in stereotaxic coordinates. San Diego: Academic Press; 1998.

18. Carvalho PG, Branco LG, Panissi CR. Involvement of the heme oxygenase-carbon monoxide-cGMP pathway in the nociception induced by acute painful stimulus in rats. Brain Res 2011; 1385: 107-113, doi: 10.1016/j.brainres.2011.02. 044.

19. Morato S, Brandão ML. Paradoxical increase of exploratory behavior in the elevated plus-maze by rats exposed to two kinds of aversive stimuli. Braz J Med Biol Res 1997; 30: 1113-1120, doi: 10.1590/S0100-879X1997000900010.

20. Pellow S, Chopin P, File SE, Briley M. Validation of open: closed arm entries in an elevated plus-maze as a measure of anxiety in the rat. J Neurosci Methods 1985; 14: 149-167, doi: 10.1016/0165-0270(85)90031-7.

21. Bourin $M$, Hascoet $M$. The mouse light/dark box test. Eur J Pharmacol 2003; 463: 55-65, doi: 10.1016/S00142999(03)01274-3.

22. Xu ZQ, Pieribone VA, Zhang X, Grillner S, Hokfelt T. A functional role for nitric oxide in locus coeruleus: immunohistochemical 
and electrophysiological studies. Exp Brain Res 1994; 98: 75-83, doi: 10.1007/BF00229111.

23. Crow TJ, Deakin JF, File SE, Longden A, Wendlandt S. The locus coeruleus noradrenergic system - evidence against a role in attention, habituation, anxiety and motor activity. Brain Res 1978; 155: 249-261, doi: 10.1016/0006-8993(78) 91021-1.

24. Rasmussen K, Jacobs BL. Single unit activity of locus coeruleus neurons in the freely moving cat. II. Conditioning and pharmacologic studies. Brain Res 1986; 371: 335-344.

25. Pirnik Z, Mravec B, Kubovcakova L, Mikkelsen JD, Kiss A. Hypertonic saline and immobilization induce Fos expression in mouse brain catecholaminergic cell groups: colocalization with tyrosine hydroxylase and neuropeptide Y. Ann N Y Acad Sci 2004; 1018: 398-404, doi: 10.1196/annals.1296.049.

26. Carrasco GA, Van de Kar LD. Neuroendocrine pharmacology of stress. Eur J Pharmacol 2003; 463: 235-272.

27. Tanaka M, Kohno Y, Nakagawa R, Ida Y, Takeda S, Nagasaki N, et al. Regional characteristics of stress-induced increases in brain noradrenaline release in rats. Pharmacol Biochem Behav 1983; 19: 543-547, doi: 10.1016/00913057(83)90132-6.

28. Graeff FG, Silveira MC, Nogueira RL, Audi EA, Oliveira RM. Role of the amygdala and periaqueductal gray in anxiety and panic. Behav Brain Res 1993; 58: 123-131, doi: 10.1016/ 0166-4328(93)90097-A.

29. Khoshbouei H, Cecchi M, Dove S, Javors M, Morilak DA. Behavioral reactivity to stress: amplification of stressinduced noradrenergic activation elicits a galanin-mediated anxiolytic effect in central amygdala. Pharmacol Biochem Behav 2002; 71: 407-417, doi: 10.1016/S0091-3057(01) 00683-9.

30. Charney DS. Neuroanatomical circuits modulating fear and anxiety behaviors. Acta Psychiatr Scand Suppl 2003; 38-50, doi: 10.1034/j.1600-0447.108.s417.3.x.

31. Hascoet M, Bourin M. A new approach to the light/dark test procedure in mice. Pharmacol Biochem Behav 1998; 60: 645-653, doi: 10.1016/S0091-3057(98)00031-8.
32. Pacak K, Palkovits M. Stressor specificity of central neuroendocrine responses: implications for stress-related disorders. Endocr Rev 2001; 22: 502-548, doi: 10.1210/ edrv.22.4.0436.

33. Ziegler DR, Cass WA, Herman JP. Excitatory influence of the locus coeruleus in hypothalamic-pituitary-adrenocortical axis responses to stress. J Neuroendocrinol 1999; 11: 361-369, doi: 10.1046/j.1365-2826.1999.00337.x.

34. Radley JJ, Williams B, Sawchenko PE. Noradrenergic innervation of the dorsal medial prefrontal cortex modulates hypothalamo-pituitary-adrenal responses to acute emotional stress. J Neurosci 2008; 28: 5806-5816, doi: 10.1523/ JNEUROSCI.0552-08.2008.

35. McGaugh JL. The amygdala modulates the consolidation of memories of emotionally arousing experiences. Annu Rev Neurosci 2004; 27: 1-28, doi: 10.1146/annurev.neuro.27. 070203.144157 .

36. Valizadegan F, Oryan S, Nasehi M, Zarrindast MR. Interaction between morphine and noradrenergic system of basolateral amygdala on anxiety and memory in the elevated plus-maze test based on a test-retest paradigm. Arch Iran Med 2013; 16: 281-287.

37. Redmond DE Jr, Huang YH, Snyder DR, Maas JW. Behavioral effects of stimulation of the nucleus locus coeruleus in the stump-tailed monkey Macaca arctoides. Brain Res 1976; 116: 502-510, doi: 10.1016/0006-8993(76)90498-4.

38. Mason ST, Fibiger HC. Current concepts. I. Anxiety: the locus coeruleus disconnection. Life Sci 1979; 25: 2141-2147, doi: 10.1016/0024-3205(79)90086-9.

39. Lapiz MD, Mateo Y, Durkin S, Parker T, Marsden CA. Effects of central noradrenaline depletion by the selective neurotoxin DSP-4 on the behaviour of the isolated rat in the elevated plus maze and water maze. Psychopharmacology 2001; 155: 251-259, doi: 10.1007/s002130100702.

40. Weiss JM, Stout JC, Aaron MF, Quan N, Owens MJ, Butler PD, et al. Depression and anxiety: role of the locus coeruleus and corticotropin-releasing factor. Brain Res Bull 1994; 35: 561-572, doi: 10.1016/0361-9230(94)90170-8. 